\title{
CHITOSAN NANOPARTICLES AND THEIR APPLICATIONS IN DRUG DELIVERY: A REVIEW
}

\author{
${ }^{1}$ Sharare Najafi, ${ }^{1}$ Zahra Pazhouhnia, ${ }^{1}$ Omid Ahmadi, \\ ${ }^{2}$ Aydin Berenjian and ${ }^{1}$ Hoda Jafarizadeh-Malmiri \\ ${ }^{1}$ Department of Chemical Engineering, Sahand University of Technology, Tabriz, Iran \\ ${ }^{2}$ School of Engineering, Faculty of Science and Engineering, University of Waikato, \\ Private Bag 3105, Hamilton 3240, New Zealand
}

Received 2014-08-24; Revised 2014-09-09; Accepted 2014-11-01

Funding: Food Engineering Research Institute of Sahand University of Technology

Competing Interests: The authors have declared that no competing interests exist

\begin{abstract}
Chitosan is one of the most popular materials in the field of drug delivery due to its adhesivity and biodegradability. Different types of Chitosan based drug carriers have been conceived for various administration routes, such as oral, nasal, transdermal, parenteral, vaginal, cervical and rectal due to its biocompatibility and biodegradability. Chitosan Nanoparticles (NPs) have long been studied as carriers for systemic and targeted drug delivery. Considerable research efforts have been directed towards developing safe and efficient Chitosan NPs-based particulate drug delivery systems. The results indicated that chemical modification of Chitosan NPs can improve their targeting and bioavailability. The main objectives of this review are to study the Chitosan NPs, their preparation methods, characteristics and modification and finally to evaluate their potential applications in drug delivery systems.
\end{abstract}

Keywords: Chitosan, Nanoparticles, Drug Delivery, Chemical Modification, Application

\section{INTRODUCTION}

Drugs have long been used to improve health and extend lives. Drug delivery systems are engineered technologies for the targeted delivery and/or controlled release of therapeutic agents (Shaik et al., 2012). Carriermediated drug delivery has emerged as a powerful methodology for the treatment of various pathologies. Nanosized particles are sophisticated technologies that were developed to answer specific demands in the field of drug delivery, namely addressing the limitations posed by the administration of a new generation of low molecular weight drugs and biomacromolecules (Rodrigues et al., 2012). For example, several therapeutic peptides and proteins represent a rapidly growing part of marketed drugs which have several drawbacks that hinder their therapeutic application.
These are undesirable physico-chemical properties, such as variable solubility, low bioavailability and limited stability (Antosova et al., 2009).

Various nanoforms have been investigated as drug delivery systems, from biological substances (albumin, gelatin and phospholipids), to chemical substances (polymers and solid metal-containing Nanoparticles (NPs) (Bamrungsap et al., 2012). NPs are taken up by cells more efficiently than larger macromolecules; therefore, they could be used as effective transport and delivery systems. NPs can be used in targeted drug delivery to improve the uptake of poorly soluble drugs and improve drug bioavailability (Suri et al., 2007).

Biodegradable polymeric NPs have attracted prominent interest in the past few decades as a novel drug carrier due to their longer half-life and greater drug entrapment efficiency (Katas et al., 2012). Furthermore, 
the polymeric NPs due to their nanoscale particle size have embraced the site-specific targeting and tend to permeate deeply into the skin substructures. Moreover, the biodegradable NPs could protect the drugs used in oral administration (e.g., hydrocortisone) from the harsh environmental conditions and genes from degradation in biological media. These NPs can also prolong the duration of drug mucoadhesion at target tissues (Katas et al., 2012).

Chitosan due to its unique physico-chemical and biological properties is an attractive material for use in various applications. Chitosan is an effective biodegradable polymer for biomedical applications due to its biocompatibility, biodegradability and low-toxicity. It also has antimicrobial activity and low immunogenicity, which clearly points to an immense potential for future development (Jafarizadeh-Malmiri et al., 2012; Vaghari et al., 2013). Chitosan nanoparticles due to their small size and large surface area to volume ratio have special characteristics, which make them favourable carriers for different drugs specially hydrophobic drugs in cancer drug delivery application (Rajan and Raj, 2013). This review emphasizes different aspects of Chitosan based nanomaterials, including their preparation, characterization and application in drug delivery systems. This review also investigates modification and toxicity of Chitosan NPs.

\section{NANOTECHNOLOGY IN DRUG DELIVERY}

Delivery of the therapeutic compounds to the target site is a major problem in treatment of many diseases. The efficacy of many drugs is often limited by their potential to reach the site of therapeutic action. In most cases (conventional dosage forms), only a small amount of administered dose reaches the target site like cancer cells, while the majority of the drug distributes throughout the rest of the body in accordance with its physico-chemical and biochemical properties (Tiyaboonchai, 2003; Patel et al., 2010).

Incorporation of an existing medicine into a drug delivery system can significantly improve its performance in terms of efficacy, safety and improved patient compliance. In drug delivery system, a drug is transported to the place of action, hence, its influence on vital tissues and undesirable side effects can be minimized. Furthermore, accumulation of therapeutic compounds in the target site increases which in turn, decreases the required doses of drugs (Bamrungsap et al., 2012).

Reducing the materials particle size is an effective method to improve their properties. This is because nanoparticles have a proportionally larger surface area than naked compounds and consequently more surface atoms than their microscale counterparts, which in turn affects their physico-chemical, optical, catalytic and other reactive properties (Suri et al., 2007).

NPs are solid colloidal particles which consist of macromolecular materials and can be used therapeutically as adjuvant in vaccines or drug carriers in which the active ingredient is dissolved, entrapped, encapsulated, adsorbed or chemically attached (Tiyaboonchai, 2003). Polymeric NPs depending on their preparation methods can be divided into two types namely: Nanospheres and nanocapsules. Nanospheres have a monolithic-type structure (matrix) in which drugs are dispersed or adsorbed onto their surfaces, while nanocapsules exhibit a membrane-wall structure and drugs are entrapped in the core or adsorbed onto their exterior Fig. 1.

Nanomedicine, has incredible potential for revolutionizing the therapeutics and diagnostics under the premise of developing ingenious nanodevices. Drug delivery nanosystems constitute a significant portion of nanomedicine. However, in drug delivery, nanotechnology is just beginning to make an impact (Park et al., 2007; Misra et al., 2010).

The main problems with current drug delivery systems are the low drug loading capacity, low loading efficiency and poor ability to control the size distribution. Utilizing nanotechnologies, such as nanopatterning, could allow manufacturing of nanocarriers with high loading efficiency and highly homogeneous particle sizes (Park et al., 2007). In fact, due to high surface to volume ratio of NPs, drugs can be easily conjugated with nanocarriers via electrostatic and hydrophobic interactions and hydrogen bonding (Tiyaboonchai, 2003).

Nanocarriers with optimized physicochemical and biological properties are taken up by cells more easily than larger molecules, so they can be successfully used as delivery tools for currently available therapeutics and drugs. Liposomes, solid lipids NPs, dendrimers, polymeric NPs, silicon or carbon materials and magnetic NPs are the examples of nanocarriers that have been tested as drug delivery systems (Wilczewska et al., 2012).

\subsection{Chitosan NPs}

Chitosan, as a derivative of chitin, is an attractive natural biopolymer from renewable resources with the presence of reactive amino and hydroxyl functional groups in its structure Fig. 2. This low-cost biopolymer is a cationic material that has attracted attention in various scientific and engineering processes due to its excellent biocompatibility, non-toxicity and chemical and thermal stability (Jafarizadeh-Malmiri et al., 2012; Vaghari et al., 2013). 


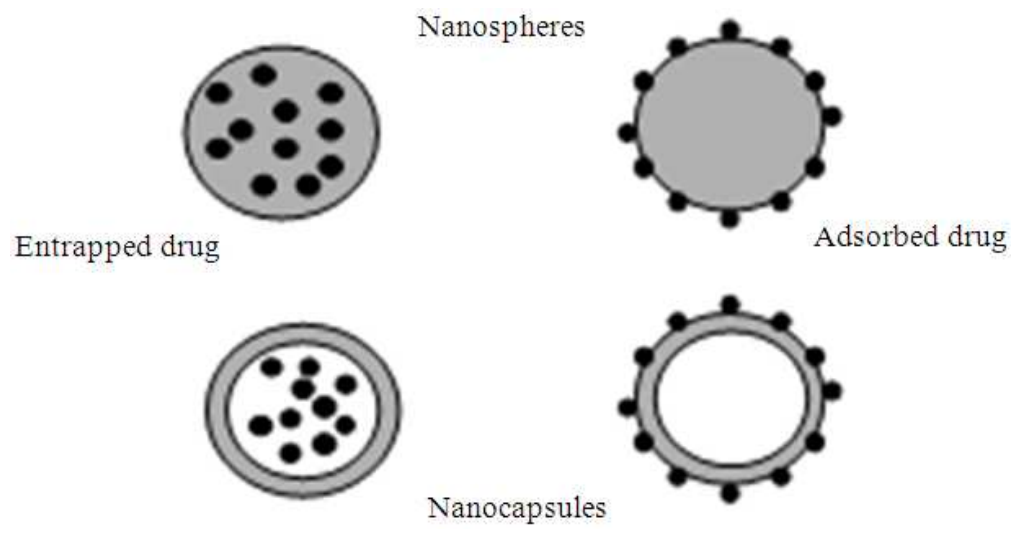

Fig. 1. Various types of drug loaded NPs (Tiyaboonchai, 2003)

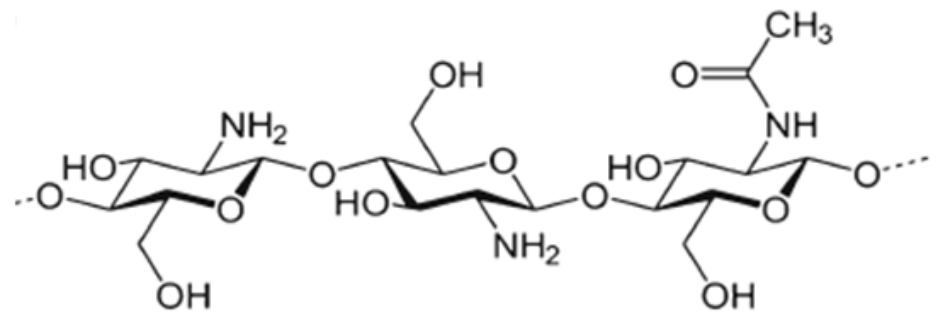

Fig. 2. Chemical structure of Chitosan (Jafarizadeh-Malmiri et al., 2012)

Chitosan NPs due to their huge surface to volume ratio as compared to the Chitosan in its bulk form, have outstanding physico-chemical, antimicrobial and biological properties. These factors make them a superior environmentally friendly material and they possess bioactivity that does not harm humans. Due to these unique properties, Chitosan NPs are being used in a variety of different products and applications, ranging from pharmaceutical, drug delivery, tissue engineering and food packaging to biosensing, enzymes immobilization, fuel cell manufacturing and waste water treatment (Jafarizadeh-Malmiri et al., 2012).

Several methods have been used to prepare Chitosan nanoparticulate. The selection of any of these methods depends on the shape and particle size requirements. Emulsion cross linking, emulsion-droplet coalescence, coacervation/precipitation, ionotropic gelation, reverse micelles, template polymerization and self-assembly polyelectrolytes are the main Chitosan nanoparticles preparation methods (Jafarizadeh-Malmiri et al., 2012). The most widely developed methods are ionotropic gelation and self-assembling polyelectrolytes. These methods offer many advantages such as simple and mild preparation method without the use of organic solvent or high shear force. Thus, they would be used in broad range of drugs including macromolecules and anticancer, i.e., Paclitaxel which notorious as labile drugs (Tiyaboonchai, 2003; Yang et al., 2009).

\subsection{Drug Loading Into Chitosan NPs}

Drug loading in NPs systems can be done by two methods, i.e., during the preparation of particles (incorporation) and after the formation of particles (incubation). In this method (incubation), drug is physically embedded into the matrix or adsorbed onto the surface. While, maximum drug loading can be achieved by incorporating the drug during the formation of particles, it may get affected by the process parameters such as method of NPs preparation which in turn, influences their shape and size and presence of additives. (Agnihorti et al., 2004). The additives can act as targeting ligands and are conjugated at the surface of the NPs for binding to appropriate receptors expressed at the target site (Manchun et al., 2012).

Applications of Chitosan NPs in the field of drug delivery have been mostly focused on the production of carriers which in turn improve the performance and effectiveness of encapsulated molecules, either macromolecules or low molecular weight drugs (Andrade et al., 2011; Dash et al., 2011; Wang et al., 2011). NPs, as solid continuous matrixes, are the most usual carriers with a Chitosan-based composition, but 
nanocapsules have also been reported (Prego et al., 2005; Park et al., 2010). Chitosan NPs have gained more attention as drug delivery carriers because of their better stability, low toxicity, simple and mild preparation method and providing versatile routes of administration (Rajan and Raj, 2013). Gnanadhas et al. (2013) successfully employed Chitosan-dextran sulphate nanocapsules containing ciprofloxacin for efficient targeting and killing of the intracellular pathogen at a dosage significantly lower than that of the free antibiotic. The increased retention time of ciprofloxacin in the blood and organs when it was delivered by Chitosan-dextran sulphate nanocapsules compared with the conventional routes of administration may be the reason underlying the requirement for a reduced dosage and frequency of antibiotic administration. Importantly, many studies report the use of Chitosan NPs as a coating material, instead of being a part of the matrix of the system, which is a relevant recognition of Chitosan's appealing properties to modify the surface properties of the core structure, either to improve the pattern of interaction with surrounding structures or to improve the biodegradation profile (Fonte et al., 2012; Grenha, 2012). In fact, Chitosan has good film forming property and hence, it can also be used as a coating material in drug delivery applications. Some of the advantages of drug coating using Chitosan NPs are improvement of drug payloads, bioadhesive property and prolonged drug release properties over the uncoated drugs (Agnihorti et al., 2004).

In general, the factors found to affect NPs formation including particle size and surface charge are molecular weight and degree of deacetylation of Chitosan. The entrapment efficiency is found to be dependent on the $\mathrm{pKa}$ and solubility of entrapped drugs. The drug is mostly found to be associated with Chitosan NPs via electrostatic interaction, hydrogen bonding and hydrophobic interaction (Tiyaboonchai, 2003).

\subsection{Chitosan NPs Applications in Drug Delivery}

Chitosan is one of the most popular materials in the field of drug delivery and is, by far, the most applied natural polymer. Its attractiveness relies on very interesting structural and biological properties, which include the cationic character and the solubility in aqueous medium (Rodrigues et al., 2012).

The adhesivity and biodegradability of Chitosan are important for its use in drug delivery systems. These properties are a result of the proper structure of the polysaccharide, which is composed of repeating alternated units of $\mathrm{N}$-acetylglucosamine and $\mathrm{D}$ glucosamine, linked by $\beta-(1-4)$ glycosidic bonds, as clearly observed in Fig. 1. The amino and carboxyl groups in the Chitosan molecule can be combined with glycoprotein in mucus to form a hydrogen bond, leading to an adhesive effect (Wang et al., 2011).

Chitosan is degraded mainly by chemical process and enzyme catalysis; the latter is the major in vivo process. The higher the degree of deacetylation, the greater the degradation rate. Degradation by enzyme catalysis also depends on the availability of Chitosan's amino group (Wang et al., 2011).

As a new drug delivery system, Chitosan NPs have attracted increasing attention for their wide applications in, for example, loading protein drugs, gene drugs and anticancer chemical drugs and via various routes of administration including oral, nasal, intravenous and ocular (Agnihorti et al., 2004).

As compared to Chitosan, its NPs can be administered intravenously due to the small diameter of the blood capillary (approximately $4 \mu \mathrm{m}$ ). The biodistribution of NPs can vary depending on the size, surface charge and hydrophobicity of the administered particles. Particles greater than $100 \mathrm{~nm}$ in diameter are rapidly taken up by the reticuloendothelial system in the liver, spleen, lung and bone marrow, while smaller-sized particles tend to have a prolonged circulation time. Negatively-charged particles are eliminated faster than positively-charged or neutral particles (Tiyaboonchai, 2003).

Therefore, drug-loaded delivery systems based on Chitosan NPs due to their small size and positive charge offer more interesting features. These systems can also be formed in aqueous solution without the use of organic solvent, surfactant and high shear. In addition, the Chitosan NPs are positively charged which can improve the site-specific targeting due to the strong affinity towards negatively charged biological membranes. Due to hydrophobicity of Chitosan, the hydrophilic drugs can be effectively encapsulated into the Chitosan NPs (Rajan and Raj, 2013).

Some of the important applications of Chitosan NPs in pharmaceutical and biomedical are related to colon targeted drug delivery (i.e., metronidazole), mucosal delivery (i.e., insulin), cancer therapy (i.e., paclitaxel), gene delivery (i.e., multidrug resistance gene, MDR1), topical delivery and ocular delivery (i.e., flurbiprofen) (Yang et al., 2009; Rajan and Raj, 2013).

Due to good bioadhesive property and ability to sustain the release of the active constituents, Chitosan NPs has been used in topical delivery systems. In gene delivery, Chitosan NPs can interact ionically with the negatively charged DNA and form polyelectrolyte complexes. In these complexes, DNA becomes better protected against 
nuclease degradation leading to better transfection efficiency (Agnihorti et al., 2004). The critical bottleneck of conventional cancer chemotherapeutics includes high toxicity of most anticancer drugs due to indiscriminate distribution of drugs towards disease and healthy cells following systemic administration. In addition, anticancer drugs often suffer from poor solubility in water and require organic solvents or detergents, resulting in undesirable side effects such as venous irritation and respiratory distress. Encapsulation a large quantity of anticancer drugs using Chitosan NPs is indispensable for successful cancer therapy (Patel et al., 2010). In addition, the positive charge of Chitosan NPs have selective adsorption and neutralizing effects on the tumor cell surface. As a drug carrier, it has a targeting function to liver, spleen, lung and colon (Wang et al., 2011). Mucosal surfaces such as nasal, peroral and pulmonary are receiving a great deal of attention as alternative routes of systemic administration. Chitosan NPs have mucoadhesive properties and it seems particularly useful to formulate the bioadhesive dosage forms for mucosal administration (ocular, nasal, buccal, gastro-enteric and vaginal-uterine therapy) (Patel et al., 2010). Chitosan NPs are also a promising polymer for colon drug delivery since they can be biodegraded by the colonic bacterial flora and they have a mucoadhesive property. Chitosan NPs also can load other drugs including antivirus drugs (i.e., diammonium glycyrrhizinate) and hormone drug (i.e., insulin) (Patel et al., 2010; Rajan and Raj, 2013).

Drug-loaded Chitosan NPs are decomposed into free Chitosan and drug in vivo. Drugs enter cell and targeted tissues to generate therapeutic effects. Chitosan is mainly degraded under catalysis of lysozyme and bacterial enzyme in the colon. The Chitosan absorbed into the blood is cleared by the kidney and the rest is discharged through excrement. Degree of deacetylation, as well as molecular weight, also influences degradation rate and degree of Chitosan in vivo (Wang et al., 2011). Table 1 indicates the main features of Chitosan NPs used for delivery of some therapeutic agents.

\subsection{Modification of Chitosan NPs to Improve their Targeting and Bioavailability}

Modification of Chitosan in order to improve its targeting and bioavailability is an important process. The primary hydroxyl and amine groups located on the backbone of Chitosan allow for chemical modification to control its physical properties (Patel et al., 2010). Modified Chitosan NPs are characterized by $\mathrm{pH}$ sensitivity, thermo sensitivity and targeting accuracy.
A pH-sensitive nanocarrier is a drug delivery system that increases drug release by changing carrier properties under a certain acid-base environment in vivo and targets the lesion tissue. It can be obtained in Chitosan NPs through chemical modification. Research has shown that Chitosan NPs prepared with sodium tripolyphosphate and glycidoxypropyltrimethoxysilane cross-linking were $\mathrm{pH}$ sensitive. With antihuman IgG antibody as a model protein drug, the release of antibody was increased from $5.6 \%$ to $50 \%$ when solution $\mathrm{pH}$ was adjusted from 7.4 to 6.0. Therefore, Chitosan NPs prepared by two-step cross-linking are potential $\mathrm{pH}$ sensitive drug carriers (Pan et al., 2009). Other research demonstrated that Camptothecine-loaded N-isopropylacrylamide-Chitosan NPs were sensitive to $\mathrm{pH}$. The results show that NPs were most sensitive to tumor $\mathrm{pH}$ when the charge ratio of $\mathrm{N}$ isopropylacrylamide: Chitosan was $4: 1$. At a $\mathrm{pH}$ of 6.8 , cumulative release rate of Camptothecine was highest and cell toxicity was significantly stronger. Cell toxicity was minimal at a $\mathrm{pH}$ of 7.4. Thus, N-isopropylacrylamideChitosan NPs have good potential for use as a targeted anticancer drug carrier (Fan et al., 2008).

Drug release is also regulated by structural change of thermosensitive drug carriers at different temperatures. Poly (N-isopropylacrylamide) is a well-known thermosensitive polymer widely used in drug carriers (Chung et al., 1996). Research has indicated that Chitosan-polyvinylcaprolactan graft copolymer NPs were sensitive to temperature, with a critical solution temperature at $38^{\circ} \mathrm{C}$. In this study 5-fluorouracil was a model drug and the drug release mainly occurred above $38^{\circ} \mathrm{C}$ with high toxicity to tumor cells but low toxicity to normal cells (Rejinold et al., 2011).

Active targeting can be obtained in Chitosan NPs through chemical modification, so as to make the drug identify the target accurately. It was shown that Chitosan NPs modified by glycyrrhizic acid, strengthened the active liver-targeting delivery of drug-loaded carriers through the mediation of glycyrrhizic acid due to glycyrrhizic acid binding on the surface of liver parenchyma (Huang et al., 2008). Oral administration of insulin remains a significant challenge because it is susceptible to hydrolysis and digestion by the acids and enzymes in the gastrointestinal tract. Also bioavailability of insulin is very low due to poor membrane permeability. One possibility to improve the gastrointestinal uptake of poorly absorbed oral drugs such as insulin is to bind to colloidal particles which can protect labile molecules from degradation in the gastrointestinal tract and promote the transport of in systemic circulation (Bhumkar et al., 2007). Table 2 summarizes the main features of modified Chitosan NPs used for delivery of insulin. 
Table 1. Chitosan NPs as carriers of some therapeutic agents

\begin{tabular}{|c|c|c|}
\hline Therapeutic agent & Main features of the formulation or pharmacological effect & References \\
\hline Insulin & $\begin{array}{l}\text { Improved the in vivo intestinal absorption } \\
\text { insulin bioavailability }\end{array}$ & (Pan et al., 2002) \\
\hline Insulin & $\begin{array}{l}\text { Altered } \mathrm{pH} \text { of the formulation } \\
\text { Enhanced interaction with the intestinal epithelium }\end{array}$ & (Ma et al., 2005) \\
\hline Ammonium glycyrrhizinate & Initial burst effect followed by continuous release of drug & (Wu et al., 2005) \\
\hline Pramipexole hydrochloride & $\begin{array}{l}\text { Enhanced mucoadhesive properties; sustained } \\
\text { release of drug in simulated intestinal fluid }\end{array}$ & (Papadimitriou et al., 2008) \\
\hline Ciprofloxacin hydrochloride & Controlled drug release & (Jain and Banerjee, 2008) \\
\hline $\begin{array}{l}\text { Streptomycin, Gentamicin, } \\
\text { Tobramycin }\end{array}$ & Controlled drug release & (Lu et al., 2009) \\
\hline Catechin & $\begin{array}{l}\text { High encapsulation efficiency } \\
\text { Controlled drug release }\end{array}$ & (Dudhani and Kosarajua, 2010) \\
\hline
\end{tabular}

$\underline{\text { Table 2. Derivatives of Chitosan as nanocarriers for delivery of insulin }}$

\begin{tabular}{lll}
\hline Chitosan derivative & $\begin{array}{l}\text { Main features of the formulation or } \\
\text { pharmacological effect }\end{array}$ & References \\
\hline Chitosan and methyl & High encapsulation efficiency & (Qian et al., 2006) \\
Methacrylate & Controlled drug release & (Jintapattanakit et al., 2007) \\
Trimethyl Chitosan & High insulin association efficiency & (Krauland and Alonso, 2007) \\
Chitosan cyclodextrin & Delayed release profile & (Bayat et al., 2008) \\
Dimethyl-ethyl Chitosan & Enhanced colonic absorption of insulin & (Bayat et al., 2008) \\
Tri-ethyl Chitosan & Enhanced colonic absorption of insulin & (Fan et al., 2008) \\
Chitosan and poly $(N$-isopropylacrylamide) & pH-dependent release of Camptothecin & (Sadeghi et al., 2008) \\
Diethylmethyl Chitosan & Better insulin loading & (Cui et al., 2009) \\
Chitosan and poly (methyl methacrylate) & pH-dependent release & (Rekha and Sharma, 2009) \\
Lauryl succinyl Chitosan & Improved mucoadhesive property & (Sandri et al., 2010) \\
& Enhanced paracellular permeation & Reduced blood sugar level \\
N-Trimethyl Chitosan & High mucoadhesive property & (Sonaje et al., 2010) \\
\end{tabular}

\section{TOXICITY OF CHITOSAN NPS}

Two of the most critical features of drug delivery systems concern their biodegradability and biocompatibility, which are mandatory requisites for acceptance by the regulatory agencies. Biodegradability becomes a crucial feature when considering acute and long-term toxicity, as nondegradable materials may accumulate in organs or even intracellularly (Rodríguez et al., 2012). Chitosan is approved by food and drug Administration (USA) for wound dressing and is generally regarded as a non-toxic and biocompatible polymer (Chaudhury and Das, 2011). However, molecular weight and degree of deacetylation of Chitosan influences its toxicity. Chitosan is degraded mainly by chemical process and enzyme catalysis. The higher the degree of deacetylation, the greater the degradation rate. Degradation by enzyme catalysis also depends on the availability of Chitosan's amino group
(Wang et al., 2011). Chitosan is mainly degraded under catalysis of lysozyme and bacterial enzyme in the colon. The Chitosan absorbed into blood is cleared by the kidney and the rest is discharged through excrement. Injection of excessive Chitosan may cause death due to blood coagulation (Yang et al., 2007). It is well-known that the pharmacokinetic properties of a drug change drastically when loaded in a nanoparticulate system due to the size, charge and surface modifications of the NPs (Chaudhury and Das, 2011). Due to the small size of Chitosan NPs, they are capable of passing through biological barriers in vivo (such as the blood-brain barrier) and delivering drugs to the lesion site to enhance efficacy. Under the action of enzymes in vivo, Chitosan NPs can produce water and carbon dioxide without adverse effects (Wang et al., 2011). Recently, few studies have been done to assess the toxicity of Chitosan NPs upon parenteral and oral administration (Carreno and Duncan, 1997; Agnihorti et al., 2004; Zhang et al., 
2008; Dube et al., 2010). The results indicated that Chitosan NPs are not toxic and Chitosan is proven to be safe in rats up $10 \%$ in the diet.

\section{CONCLUSION}

Many drugs have problems of poor stability, water insolubility, low selectivity, high toxicity, side effects and so on. Clinically useful drug delivery systems need also to deliver a certain amount of a drug that can be therapeutically effective over an extended period of time. Such requirements can be met by the nano scale drug delivery systems. Chitosan NPs are good drug carriers because of their good biocompatibility, biodegradability and non toxicity and can be readily modified. As a new drug delivery system, they have attracted increasing attention for their wide applications. Chitosan nanoparticles are now being modified for sustained/ controlled release and targeting. Although Chitosan NPs can be easily modified to carrier, coat and encapsulate hydrophobic drugs, further investigation is required on the biocompatibility of modified Chitosan NPs and its derivatives.

\section{ACKNOWLEDGEMENT}

The work was supported and funded by the Food Engineering Research Institute of Sahand University of Technology.

\section{AUTHOR'S CONTRIBUTIONS}

Sharareh Najafi, Zahra Pazhouhnia and Omid Ahmadi prepared the manuscript. Aydin Berenjian contributed helpful discussion during the preparation of manuscript. Hoda Jafarizadeh-Malmiri revised and approved the final version of the manuscript.

\section{REFERENCES}

Agnihorti, S.A., N.N. Mallikarjuna and T.M. Aminabhavi, 2004. Recent advances on Chitosanbased micro-and nanoparticles in drug delivery. J. Controlled Release, 100: 5-28. DOI: 10.1016/j.jconrel.2004.08.010

Andrade, F., F. Antunes, A. Nascimento, S. Da Silva and J. das Neves et al., 2011. Chitosan formulations as carriers for therapeutic proteins. Current Drug Discovery Tech., 8: 157-172. DOI: $10.2174 / 157016311796799035$
Antosova, Z., M. Mackova, V. Kral and T. Macek, 2009. Therapeutic application of peptides and proteins: Parenteral forever? Trends in Biotech., 27: 628-635. DOI: 10.1016/j.tibtech.2009.07.009

Bamrungsap, S., Z. Zhao, T. Chen, L. Wang and C. Li et al., 2012. Nanotechnology in therapeutics. Nanomedicine, 7: 1253-1271. DOI: 10.2217/nnm.12.87

Bayat, A., F.A. Dorkoosh, A.R. Dehpour, L. Moezi and B. Larijani et al., 2008. Nanoparticles of quaternized Chitosan derivatives as a carrier for colon delivery of insulin: Ex vivo and in vivo studies. Int. J. Pharmaceutics, 35: 259-266. DOI: 10.1016/j.ijpharm.2007.12.037

Bhumkar, D.R., H.M. Joshi, M. Sastry and V.B. Pokharkar, 2007. Chitosan reduced gold nanoparticles as novel carriers for transmucosal delivery of insulin. Pharmaceutical Res., 4: 14151426. DOI: 10.1007/s11095-007-9257-9

Carreno, G.B. and R. Duncan, 1997. Evaluation of the biological properties of soluble Chitosan and Chitosan microspheres. Int. J. Pharmaceutics, 148: 231-240. DOI: 10.1016/S0378-5173(96)04847-8

Chaudhury, A. and S. Das, 2011. Recent advancement of Chitosan-based nanoparticles for oral controlled. Am. Associat. Pharmaceutical Sci. Pharmscitech, 12: 10-20. DOI: 10.1208/s12249-010-9561-2

Chung, J., M. Yokoyama, M. Yamato, T. Aoyagi and Y. Sakurai et al., 1996. Thermo-responsive drug delivery from polymeric micelles constructed using block copolymers of poly ( $\mathrm{N}$-isopropylacrylamide) and poly (butylmethacrylate). J. Controlled Release, 62: 115-127. DOI: 10.1016/S0168-3659(99)00029-2

Cui, F., F. Qian, Z. Zhao, L. Yin and C. Tang et al., 2009. Preparation, characterization and oral delivery of insulin loaded carboxy-lated Chitosan grafted poly (methyl methacrylate) nanoparticles. Biomacromolecules, 10: 1253-1258. DOI: $10.1021 / \mathrm{bm} 900035 \mathrm{u}$

Dash, M., F. Chiellini, R.M. Ottenbrite and E. Chiellini 2011. Chitosan-A versatile semi-synthetic polymer in biomedical applications. Progress Polymer Sci., 36: 981-1014. DOI: 10.1016/j.progpolymsci.2011.02.001

Dube, A., J.A. Nicolazzo and I. Larson, 2010. Chitosan nanoparticles enhance the intestinal absorption of the green tea catechins (+)-catechin and (-)epigallocatechin gallate. Eur. J. Pharmaceutical Sci., 41: 219-225. DOI: 10.1016/j.ejps.2010.06.010 
Dudhani, A.R. and S.L. Kosarajua, 2010. Bioadhesive Chitosan nanoparticles: Preparation and characterization. Carbohydrate Polymers, 81: 243251. DOI: $10.1016 /$ j.carbpol.2010.02.026

Fan, L., H. Wu, H. Zhang, F. Li and T. Yang et al., 2008. Novel super $\mathrm{pH}$-sensitive nanoparticles responsive to tumor extracellular pH. Carbohydrate Polymers, 73: 390-400. DOI: 10.1016/j.carbpol.2007.12.006

Fonte, P., F. Andrade, F. Araújo, C. Andrade and J.D. Neves et al., 2012. Chitosan-coated solid lipid nanoparticles for insulin delivery. Methods Enzymol., 508: 295-314. DOI: 10.1016/B978-0-12391860-4.00015-X

Gnanadhas, D.P., M. Ben Thomas, M. Elango and M.A.M. Raichur et al., 2013. Chitosan-dextran sulphate nanocapsule drug delivery system as an effective therapeutic against intraphagosomal pathogen Salmonella. J. Antimicrobial Chemotherapy, 68: 2576-2586. DOI: 10.1093/jac/dkt252

Grenha, A., 2012. Chitosan nanoparticles: A survey of preparation methods. J. Drug Target., 20: 291-300. DOI: 10.3109/1061186X.2011.654121

Huang, Y., A.H. Lin and X. Zhang, 2008. Targeting binding of Chitosan nanoparticles with glycyrrhizin surface modification to hepatic parenchymal cells in vitro. Traditional Chinese Drug Res. Pharmacol., 19: 495-498.

Jafarizadeh-Malmiri, H., M.A. Gaz-Jahanian and A. Berenjian, 2012. Potential applications of Chitosan nanoparticles as novel support in enzyme immobilization. Am. J. Biochem. Biotechnol., 8: 203-219. DOI: 10.3844/ajbbsp.2012.203.219

Jain, D. and R. Banerjee, 2008. Comparison of ciprofloxacin hydrochloride-loaded protein, lipid and Chitosan nanoparticles for drug delivery. J. Biomed. Materials Res. Part B: Applied Biomaterials, 86: 105-112. DOI: 10.1002/jbm.b.30994

Jintapattanakit, A., V.B. Junyaprasert, S. Mao, J. Sitterberg and U. Bakowsky et al., 2007. Peroral delivery of insulin using Chitosan derivatives: A comparative study of polyelectrolyte nanocomplexes and nanoparticles. Int. J. Pharmaceutics, 342: 240-249. DOI: 10.1016/j.ijpharm.2007.05.015

Katas, H., Z. Hussain and T.C. Ling, 2012. Chitosan nanoparticles as a percutaneous drug delivery system for hydrocortisone. J. Nanomaterials, 2012: 11-11. DOI: $10.1155 / 2012 / 372725$
Krauland, A.H. and M.J. Alonso, 2007. Chitosan/cyclodextrin nanoparticles as macromolecular drug delivery system. Int. J. Pharmaceutics, 340: 134-142. DOI: 10.1016/j.ijpharm.2007.03.005

Lu, E., S. Franzblau, H. Onyuksel and C. Popescu, 2009. Preparation of aminoglycoside-loaded Chitosan nanoparticles using dextran sulphate as a counterion. J. Microencapsulat., 26: 346-354. DOI: 10.1080/02652040802365182

Ma, Z., T.M. Lim and L.Y. Lim, 2005. Pharmacological activity of peroral Chitosan-insulin nanoparticles in diabetic rats. Int. J. Pharmaceutics, 293: 271-280. DOI: 10.1016/j.ijpharm.2004.12.025

Manchun, S., C.R. Dass and P. Sriamornsak, 2012. Targeted therapy for cancer using $\mathrm{pH}$-responsive nanocarrier systems. Life Sci., 90: 381-387. DOI: 10.1016/j.lfs.2012.01.008

Misra, R., S. Acharya and S.K. Sahoo, 2010. Cancer nanotechnology: application of nanotechnology in cancer therapy. Drug Discovery Today, 15: 842-850. DOI: 10.1016/j.drudis.2010.08.006

Pan, A., B.B. Wu and J.M. Wu, 2009. Chitosan nanoparticles cross linked by glycidoxypropyltrimethoxysilane for $\mathrm{pH}$ triggered release of protein. Chinese Chemical Lett., 20: 79-83. DOI: 10.1016/j.cclet.2008.10.012

Pan, Y., Y.J. Li, H.Y. Zhao, J.M. Zheng and H. Xu et al., 2002. Bio adhesive polysaccharide in protein delivery system: Chitosan nanoparticles improve the intestinal absorption of insulin in vivo. Int. J. Pharmaceutics, 249: 139-147. DOI: 10.1016/S03785173(02)00486-6

Papadimitriou, S., D. Bikiaris, K. Avgoustakis, E. Karavas and M. Georgarakis, 2008. Chitosan nanoparticles loaded with dorzolamide and pramipexole. Carbohydrate Polymers, 73:44-54. DOI: 10.1016/j.carbpol.2007.11.007

Park, J.H., G. Saravanakumar, K. Kim and I.C. Kwon, 2010. Targeted delivery of low molecular drugs using Chitosan and its derivatives. Adv. Drug Delivery Rev., 62: 28-41. DOI: 10.1016/j.addr.2009.10.003

Patel, M.P., R.R. Patel and J.K. Patel, 2010. Chitosan mediated targeted drug delivery system: A review. J. Pharmacy Pharmaceutical Sci., 13: 536-557.

Prego, C., D. Torres and M.J. Alonso, 2005. The potential of Chitosan for the oral administration of peptides. Expert Opinion Drug Delivery, 2: 843-854. DOI: $10.1517 / 17425247.2 .5 .843$ 
Qian, F., F. Cui, J. Ding, C. Tang and C. Yin, 2006. Chitosan graft copolymer nanoparticles for oral protein drug delivery: Preparation and characterization. Biomacromolecules, 7: 27222727. DOI: $10.1021 / \mathrm{bm} 060065 \mathrm{f}$

Rajan, M. and V. Raj, 2013. Potential drug delivery applications of Chitosan based nanomaterials. Int. Rev. Chemical Eng., 5: 145-155.

Rejinold, N.S., K.P. Chennazhi, S.V. Nair, H. Tamura and R. Jayakumar, 2011. Biode-gradable and thermo-sensitive Chitosan-g-poly (Nvinylcaprolactam) nanoparticles as a 5fluorouracil carrier. Carbohydrate Polymers, 83: 776-786. DOI: 10.1016/j.carbpol.2010.08.052

Rekha, M.R. and C.P. Sharma, 2009. Synthesis and evaluation of lauryl succinyl Chitosan particles towards oral insulin delivery and absorption. J. Controlled Release, 135: 144-1451. DOI: 10.1016/j.jconrel.2009.01.011

Rodrigues, S., M. Dionísio, C.R. López and A. Grenha, 2012. Biocompatibility of Chitosan carriers with application in drug delivery. J. Functional Biomaterials, 3: 615-641. DOI: 10.3390/jfb3030615

Rodríguez, A., Rodríguez, B., Lucas, M.P., 2012 a. Trends in numbers of petrels attracted to arti fi cial lights suggest population declines in Tenerife, Canary Islands. IBIS, 154: 167-172.

Sadeghi, A.M., F.A. Dorkoosh, M.R. Avadi, P. Saadat and M. Rafiee-Tehrani et al., 2008. Preparation, characterization and antibacterial activities of Chitosan, N-Trimethyl Chitosan (TMC) and NDiethylmethyl Chitosan (DEMC) nanoparticles loaded with insulin using both the ion tropic gelation and polyelectrolyte complexation methods. Int. J. Pharmaceutics, 355: 299-306. DOI: 10.1016/j.ijpharm.2007.11.052

Sandri, G., M.C. Bonferoni, S. Rossi, F. Ferrari and C. Boselli et al., 2010. Insulin-loaded nanoparticles based on N-trimethyl Chitosan: In vitro (Caco-2 model) and ex vivo (excised rat jejunum, duodenum and ileum) evaluation of penetration enhancement properties. Am. Associat. Pharmaceutical Sci. Pharm. Sci. Tech., 11: 362371. DOI: 10.1208/s12249-010-9390-3

Shaik, M.R., M. Korsapati and D. Panati, 2012. Polymers in controlled drug delivery systems. In. J. Pharm. Sci., 2: 112-116.
Sonaje, K., Y.J. Chen, H.L. Chen, S.P. Wey and J.H. Juang et al., 2010. Enteric-coated capsules filled with freeze-dried Chitosan/poly (gamma-glutamic acid) nanoparticles for oral insulin delivery. Biomaterials, 31: 3384-3394. DOI: 10.1016/j.biomaterials.2010.01.042

Suri, S.S., H. Fenniri and B. Singh, 2007. Nanotechnology-based drug delivery systems. J. Occupat. Med. Toxicol., 2: 1-6. DOI: 10.1186/17456673-2-1

Tiyaboonchai, W., 2003. Chitosan nanoparticles: A promising system for drug delivery. Naresuan University J., 11: 51-66.

Vaghari, H., H. Jafarizadeh-Malmiri, A. Berenjian and N. Anarjan, 2013. Recent advances in application of Chitosan in fuel cells. Sustainable Chem. Process., 1: 16-16. DOI: 10.1186/2043-7129-1-16

Wang, J.J., Z.W. Zeng, R.Z. Xiao, T. Xie and G.L. Zhou et al., 2011. Recent advances of Chitosan nanoparticles as drug carriers. Int. J. Nanomed., 6: 765-774. DOI: 10.2147/IJN.S17296

Wilczewska, A.Z., K. Niemirowicz, K.H. Markiewicz and H. Car, 2012. Nanoparticles as drug delivery systems. Pharmacolog. Reports, 64: 1020-1037. DOI: 10.1016/S1734-1140(12)70901-5

Wu, Y., W. Yang, C. Wang, J. Hu and S. Fu, 2005. Chitosan nanoparticles as a novel delivery system for ammonium glycyrrhizinate. Int. J. Pharmaceutics, 295: 235-145. DOI: 10.1016/j.ijpharm.2005.01.042

Yang, Y., Z. Wang, M. Li and S. Lu, 2009. Chitosan/pshRNA plasmid nanoparticles targeting MDR1 gene reverse paclitaxel resistance in ovarian cancer cells. J. Huazhong University Sci. Tech., 29: 239-242. DOI: 10.1007/s11596-009-0221-2

Yang, Y.M., W. Hu, X.D. Wang and X.S. Gu, 2007. The controlling biodegradation of Chitosan fibers by $\mathrm{N}$ acetylation in vitro and in vivo. J. Materials Sci. Materials Med., 18: 2117-2121. DOI: 10.1007/s10856-007-3013-X

Zhang, C., G.W. Qu, Y.J. Sun, T. Yang and Z. Yao et al., 2008. Biological evaluation of N-octyl-O-sulfate Chitosan as a new nano-carrier of intravenous drugs. Eur. J. Pharmaceutical Sci., 33: 415-423. DOI: 10.1016/j.ejps.2008.01.012 